\title{
PATRONES DE COMPORTAMIENTO DIURNO DE HUEREQUEQUE BURHINUS SUPERCILIARIS EN HÁBITATS MODIFICADOS DE LA COSTA CENTRAL DEL PERÚ
}

\author{
José IANNACONE, ${ }^{1,2}$ Wignard VILLEGAS, ${ }^{1}$ MARIANEla CALDERÓN, ${ }^{1}$ \\ JORGE HUAMÁN, ${ }^{1}$ MARIO SILVA-SANTIESTEBAN ${ }^{1}$ y LORENA ALVARIÑO ${ }^{1}$ \\ ${ }^{1}$ Laboratorio de Ecofisiología Animal. Facultad de Ciencias Naturales y Matemática. Universidad \\ Nacional Federico Villarreal. Av. Río de Chepén s/n. Urb. Villa Hermosa. Bravo Chico, El Agustino, \\ Lima, Perú. \\ ${ }^{2}$ Museo de Historia Natural. Facultad de Ciencias Biológicas. Universidad Ricardo Palma (URP). Av. \\ Benavides 5440, Santiago de Surco, Lima, Perú. \\ joseiannacone@gmail.com
}

Iannacone, J., Villegas, W., Calderón, M., Huamán, J., Silva-Santiesteban, M. \& Alvariño, L. 2012. Patrones de comportamiento diurno de Huerequeque Burhinus superciliaris en hábitats modificados de la costa central del Perú. Acta Zoológica Mexicana (n. s.), 28(3): 507-524.

RESUMEN. El objetivo de este trabajo fue estudiar los patrones de comportamiento diurno de Huerequeque Burhinus superciliaris (Tschudi, 1843) en condiciones naturales y elucidar los patrones de comportamiento para esta especie. Los datos de campo fueron obtenidos en remanentes de tierras agrícolas y hábitats modificados en un área de $350 \mathrm{~m}^{2}$ en la ecorregión central del Desierto Pacífico Costero del Perú. Este estudio se realizó de mayo a julio del 2006 totalizando 30 h de observación. Fueron detectadas 32 conductas, agrupadas en nueve categorías de comportamiento. Las tres principales categorías de agrupación de comportamiento en orden porcentual decreciente con relación al tiempo total de observación fueron: acicalamiento (49.7\%), alerta (28.3\%) y locomoción (13.5\%), y otras categorías (8.5\%). Se ha encontrado que el Huerequeque con relación al tiempo total de observación realiza principalmente las siguientes cuatro conductas: centinela $(24.8 \%)$, caminar $(10.9 \%)$, movimiento de la cola $(7.2 \%)$ y limpieza de las plumas del pecho (9.0\%). La caracterización de los patrones de comportamiento de $B$. superciliaris puede contribuir a su conservación in situ bajo el sistema nacional de áreas naturales protegidas en el Perú.

Palabras clave: Acicalamiento, Burhinus, comportamiento, etograma, locomoción.

Iannacone, J., Villegas, W., Calderón, M., Huamán, J., Silva-Santiesteban, M. \& Alvariño, L. 2012. Behavioral diurnal patterns of the Peruvian thick-knee Burhinus superciliaris in modified habitats of the central coast of Peru. Acta Zoológica Mexicana (n. s.), 28(3): 507-524.

ABSTRACT. The aim of this research was to study the behavioral diurnal patterns of the Peruvian Thick-Knee Burhinus superciliaris (Tschudi, 1843) in the wild and to elucidate behavioral patterns for this species. Field efforts were carried out in remaining agricultural lands and modified habitats in an

Recibido: 21/02/2011; aceptado: 10/07/2012. 
area of $350 \mathrm{~m}^{2}$ in central Coastal Pacific Desert of Peru. We conducted focal observations of individuals from May to July of 2006 during a total of $30 \mathrm{~h}$ of observation. A total of 32 movements were detected and grouped in nine categories of behaviors. The three main categories of behavior, in decreasing order of percentage in relation to total time of observation, were: maintenance $(49.7 \%)$, vigilance $(28.3 \%)$, locomotion $(13.5 \%)$ and other categories $(8.5 \%)$. In relation to total time of observation, the Peruvian Thick-Knee conducted the following main four movements: vigilance $(24.8 \%)$, walk (10.9\%), moving the tail $(7.2 \%)$, preening of chest feathers $(9.0 \%)$ and the rest of conducts $(48.1 \%)$. Characterization of behavior patterns of $B$. superciliaris may contribute to in situ conservation under the National System of Protected Natural Areas of Peru.

Key words: Behavior, Burhinus, ethogram, locomotion, maintenance.

\section{INTRODUCCIÓN}

E1 Huerequeque, Alcaraván Peruano o Chorlo Cabezón Burhinus superciliaris (Tschudi, 1843) (Charadriiformes: Burhinidae), es un ave gregaria principalmente crepuscular y nocturna (Salinas et al. 2003, Schulenberg et al. 2010). Presenta una talla entre 38 a $43 \mathrm{~cm}$ y se alimenta principalmente de insectos ortópteros y coleópteros (Jones 1997, Schulenberg et al. 2010). Habita en zonas áridas y semiáridas de poca vegetación a lo largo de toda la costa peruana, hasta Ecuador y el norte de Chile con una extensión de ocurrencia global estimada de $80,000 \mathrm{~km}^{2}$ (Williams 1981, Miranda et al. 2001, Salinas et al. 2003, Aguirre et al. 2006, Salinas et al. 2007, Takano \& Castro 2007). Esta especie residente es considerada rara y endémica de la costa ecuatoriana, peruana y chilena, prefiriendo áreas desérticas sin cobertura vegetal o con vegetación arbustiva y herbácea escasa como los gramadales. En el Perú, se distribuye entre los departamentos de Piura y Tacna, principalmente cerca a la costa del litoral del Pacífico bajo la influencia de la Corriente de Humboldt (Astohuamán \& Espejo 2004, Valqui 2004, PromPerú 2005, Camacho 2012). El Huerequeque se encuentra protegido por el estado peruano en diversas unidades de conservación como el Parque Nacional Cerros de Amotape, las Reservas Nacionales de Lachay, Paracas, Lagunas de Mejía, y los Refugios de vida silvestre Laquipampa y Pantanos de Villa (Pautraut \& Riveros 1998, Iannacone et al. 2010), así como en diversos humedales costeros de importancia para la conservación de aves, como Puerto Etén (Lambayeque, Perú) (Angulo-Pratolongo et al. 2010). Valqui (2004) considera que los campos abandonados con ausencia de cobertura vegetal cercanos a los humedales de Puerto Viejo en el departamento de Lima, Perú son lugares ideales para la observación del Huerequeque.

El tamaño poblacional global del Huerequeque no ha sido cuantificado (BirdLife International 2004), pero aunque no parece tener problemas de riesgo de extinción, al ser catalogada como de Preocupación menor (LC), la carencia de conocimiento de esta especie requiere de una mayor investigación (Jones 1997, Camacho 2012). Para efectuar actividades de manejo y conservación in situ en aves es necesario entre otros aspectos realizar estudios básicos de comportamiento o etogramas en condiciones 
naturales, debido a que la disposición de ánimo, la salud y la psicología de los animales se manifiestan por sus patrones motores cuantitativos que se modifican para cada especie de ave ante la presencia de diferentes impactos ambientales (Prestes 2000, Porto \& Piratelli 2005, Ibañez \& Iannacone 2011). Una de las ventajas en los estudios en condiciones ex situ es que se pueden disminuir las conductas anómalas en los animales ocasionadas por el estrés del confinamiento o cautiverio (Griggio \& Hoy 2006, Córdova-Rojas \& Iannacone 2011).

Este trabajo tiene como objetivo principal estudiar patrones del comportamiento diurno de la especie nocturna $B$. superciliaris en hábitats modificado por el hombre, en la costa central del Perú. Esta información es importante para entender el empleo del tiempo a lo largo de su ciclo diario de actividades, y es en este periodo de inactividad aparente "reposo" cuando más riesgos podría tener la especie de sufrir depredación por animales diurnos, lo cual ocasiona gastos energéticos y afecta en alguna medida sus patrones de actividad.

\section{MATERIALES Y MÉTODOS}

Área de estudio. El área de estudio fue de aproximadamente $350 \mathrm{~m}^{2}(35 \mathrm{~m} \mathrm{x} 10 \mathrm{~m})$ y se localizó en la zona noroeste en el ex Fundo Oquendo, ahora denominado Centro de Investigaciones Agroecológicas de la Universidad Nacional Federico Villarreal (CIAE-UNFV) con un área total de $6000 \mathrm{~m}^{2}$, Ventanilla, Callao, Perú. Biogeográficamente se encuentra en la Región Subtropical. Presenta un clima templado y con una temperatura promedio anual de $18{ }^{\circ} \mathrm{C}$ y $80 \%$ de humedad relativa. Las observaciones de campo se realizaron en la ecorregión central del Desierto Pacífico Costero, que es un terreno seco y abandonado, remanente de tierras agrícolas y hábitats modificados, apreciándose piedras y restos de plantas secas producto de la cosecha. En la zona noroeste del área de estudio, se apreciaron cultivos de tomate, frijol y de maíz, los que son regados con aguas residuales no tratadas (Alcantara, 2010). El CIAE se encuentra rodeado por muros de ladrillo de $3 \mathrm{~m}$ de altura y presenta bordeando la parte interna a dos $\mathrm{m}$ del muro una pista asfaltada $3 \mathrm{~m}$ de ancho que permite la movilización durante el día del personal administrativo (18 personas) que custodian el local. En la zona suroeste del CIAE se encuentra un invernadero de $10 \mathrm{~m} \times 4 \mathrm{~m}$, dos oficinas administrativas y una Planta Piloto de Conservas Pesqueras. En la parte central del CIAE se encuentran restos arqueológicos de la cultura Chillón "Huacas". En la parte oeste del CIAE se localiza un área de almacenaje de vinos y cultivo de viñedos. Se observaron un total de cuatro perros domesticados y dos especies de aves como posibles depredadores de B. superciliaris, el cernícalo Falco sparverius y la lechuza de los arenales Athene cunicularia. El ingreso al CIAE es restringido solamente a los estudiantes y docentes universitarios que visitan el CIAE ocasionalmente, para realizar algunas prácticas durante el semestre académico. 
Iannacone et al.: Comportamiento de Burhinus superciliaris

Patrones de comportamiento. El comportamiento de un grupo de aproximadamente $387 \pm 93$ (rango: 261-529) individuos por semana de B. superciliaris, fue estudiado sin distinción de sexo (ya que carecen de dimorfismo sexual, Jones 1997), durante mayo a julio del 2006, mayormente en forma semanal. La elección de estos tres meses fue realizada por ser representativa para el Perú del periodo no reproductivo que es de marzo a septiembre, y por presentarse en este periodo la mayor concentración y agregación poblacional (Camacho 2012). Las observaciones se hicieron con la ayuda de binoculares (marca Tasco ${ }^{\circledR}$ con aumentos de $8 \times 21$ ) a una distancia mayor a $15 \mathrm{~m}$ de tal manera que los observadores no fueran detectados por el ave. La vestimenta del observador fue de color marrón claro muy similar al suelo donde viven las aves y se empleó como escondite o hide, un montículo de restos de construcción de $3 \mathrm{~m}$ de largo por $2 \mathrm{~m}$ de ancho por $1.5 \mathrm{~m}$ de alto para asegurarse de no afectar el comportamiento de las aves. Un análisis previo mostró que distancias menores a 15 $\mathrm{m}$ en el hide ocasionaron un incremento en la caminata, vuelos cortos y un mayor estado de alerta (Maruyama et al. 2010). Se siguió el muestreo de comportamiento focal según el protocolo de Blumstein (2006). Se tomaron registros fotográficos (Emlen \& Wrege 2004) y se empleó una cámara fotográfica digital y una filmadora de video por un periodo de 15 min cada día y por individuo. Todos estos registros audiovisuales fueron posteriormente procesados en gabinete, y se verificó si algún comportamiento de B. superciliaris había sido pasadopor alto, para ser incluído en el etograma.

Las observaciones totalizaron $30 \mathrm{~h}$, en horas de la mañana de 7:00 a 12:00 h (21 horas: siete en mayo, siete en junio y siete en julio), por la tarde de 15:00 a 18:00 h (8 horas: tres en mayo; tres en junio y dos en julio) y por la noche de 18:00 a 19:00 (1 $\mathrm{h}$; una en julio). Las sesiones no excedieron de tres horas por día y mayormente una sesión por semana, con cinco minutos de descanso por hora de observación. En cada sesión de observación participaron cuatro observadores, quienes se turnaron de a dos en intervalos de quince minutos para determinar las conductas del Huerequeque. El estudio fue realizado con énfasis en el periodo diurno, debido a que el comportamiento de esta especie pudiera estar influenciado por presiones como depredadores diurnos y por aspectos climáticos, y para relacionarlo con otras especies nocturnas que presentan actividades diurnas sin un aparente descanso continuo (Camacho 2012). Con el fin de asegurar que el número diferente de horas de observación entre la mañana y la tarde no influenciara el resultado se realizó una comparación estadística entre el número de conductas observadas en $B$. superciliaris entre la mañana (13.8 \pm $2.6 ; \mathrm{n}=21)$ y la tarde $(13.6 \pm 2.8 ; \mathrm{n}=8)$, no observándose diferencias significativas (Homogeneidad de varianzas $\mathrm{F}=0.042, \mathrm{P}>0.05 ; \mathrm{t}=0.20, \mathrm{p}=0.83$ ). La observación cualitativa y cuantitativa se centró siempre en un número limitado de individuos que fueron de dos a tres o en uno solo por observador por periodo de 15 minutos evaluado (Maruyama et al. 2010), para detallar la actividad del ave y la elaboración de su 
etograma que es el conjunto de patrones de comportamiento de un ave (Vaz-Ferreira 1984, Rivadeneyra et al. 2000, Lahitte et al. 2002).

Se evaluaron a lo largo de un día un promedio de seis individuos y en las 30 horas un total de 173 individuos de $B$. superciliaris. Los comportamientos se registraron de acuerdo a su frecuencia y a la duración de cada tipo de comportamiento. Debido a que no se encontraron comportamientos asociados con el periodo reproductivo como cortejo o cópula, incubación o presencia de crías, nuestro estudio se realizó básicamente en la estación no reproductiva (Miranda et al. 2001, Maruyama et al. 2010, Camacho 2012).

Los comportamientos fueron definidos y agrupados en las categorías propuestas por Mikich (1991) y Prestes (2000). A partir de todas las observaciones realizadas, fueron elaborados y trazados los etogramas. Fue trazada una curva acumulativa por cada conducta nueva que se iba detectando a lo largo de cada una de las $30 \mathrm{~h} \mathrm{de} \mathrm{ob-}$ servación.

\section{RESULTADOS}

Fueron identificadas y descritas 32 conductas o movimientos, agrupadas en nueve categorías de comportamiento: locomoción $(n=5)$, acicalamiento $(n=16)$, alimentación $(\mathrm{n}=1)$, descanso $(\mathrm{n}=2)$, alerta $(\mathrm{n}=2)$, sonora $(\mathrm{n}=2)$, defecación $(\mathrm{n}=1)$, social agonística $(\mathrm{n}=1)$ y social no agonístico $(\mathrm{n}=2)$ (Cuadro 1; Figs. 1-6). Nuestros resultados muestran que el huerequeque no presenta un aparente descanso diurno o "reposo" (5.5\%).

De las nueve categorías de comportamiento asignadas se observó la siguiente secuencia en orden porcentual decreciente con relación al tiempo total de observación: acicalamiento (49.7\%), alerta (28.3\%), locomoción (13.5\%), descanso (5.5\%), comer (1.8\%), y las cuatro restantes menos del $1 \%$ cada una $(1.2 \%)$.

Se ha encontrado la siguiente secuencia en orden porcentual decreciente de las cuatro principales conductas identificadas en $B$. superciliaris con relación al tiempo total de observación: centinela, caminar, movimiento de la cola y limpieza de las plumas del pecho (Cuadro 1).

Se observaron las siguientes conductas del etograma relacionadas en secuencia: a) movimiento de la cola y sacude el cuerpo (dos eventos) (>1 min); b) erección de plumas, sacudido del cuerpo y movimiento de cola (tres eventos) (1 min); c) movimiento de la cola, erección de plumas, sacudida del cuerpo, movimiento de cola y camina (cinco eventos) (1 min); d) limpieza de las plumas del pecho, limpieza de las plumas del ala, limpieza de las plumas del dorso y recojo de cera (cuatro eventos) (3 min aproximadamente) (Fig. 7a-d).

Los resultados obtenidos de la curva de colecta nos indican que probablemente aún podrían encontrarse y elucidadas nuevas conductas de comportamiento en continuidad a las observaciones realizadas, pues no se estandarizó la curva (Fig. 8). Esto 
Cuadro 1. Etograma del Huerequeque, Burhinus superciliaris (Aves, Charadriiformes, Burhinidae).

\begin{tabular}{|c|c|c|c|c|}
\hline No. & $\begin{array}{c}\text { Conductas } \\
\text { en nueve } \\
\text { categorías de } \\
\text { comportamiento }\end{array}$ & Caracterización de cada conducta & $\begin{array}{l}\text { No. horas de } \\
\text { observación }\end{array}$ & $\begin{array}{c}\text { Tiempo } \\
\text { total de } \\
\text { observación } \\
(\%)\end{array}$ \\
\hline \multicolumn{5}{|c|}{ Acicalamiento } \\
\hline 1 & $\begin{array}{l}\text { Limpieza de las } \\
\text { plumas del dorso }\end{array}$ & $\begin{array}{l}\text { El ave voltea la cabeza hacia el dorso y } \\
\text { lubrica con la cera recogida de la cola con } \\
\text { el pico. }\end{array}$ & $1: 35$ & 5.3 \\
\hline 2 & $\begin{array}{l}\text { Limpieza de las } \\
\text { plumas de pecho }\end{array}$ & $\begin{array}{l}\text { El individuo esta de pie e inclina su } \\
\text { cabeza en dirección del pecho y dejaba } \\
\text { su saliva en las plumas y acomodaba su } \\
\text { pluma con el pico. }\end{array}$ & $2: 42$ & 9.0 \\
\hline 3 & $\begin{array}{l}\text { Limpieza de las } \\
\text { plumas de las alas }\end{array}$ & $\begin{array}{l}\text { B. superciliaris, de pie sobre el substrato } \\
\text { utiliza el pico para la limpieza de las } \\
\text { plumas de las alas. La cabeza la voltea } \\
\text { para meterla bajo el ala muchas veces } \\
\text { debajo o algunas veces por arriba de las } \\
\text { plumas con el pico. }\end{array}$ & $1: 27$ & 4.8 \\
\hline 4 & $\begin{array}{l}\text { Limpieza de las } \\
\text { plumas de las } \\
\text { axilas }\end{array}$ & $\begin{array}{l}\text { Con una de las alas entreabierta, el } \\
\text { individuo voltea la cabeza en dirección } \\
\text { de la axila, deja la saliva con el pico } \\
\text { alternadamente en cada pluma. }\end{array}$ & $0: 41$ & 2.3 \\
\hline 5 & Limpieza de pico & $\begin{array}{l}\text { El ave pasa el pico lateral y } \\
\text { alternadamente varias veces de derecha a } \\
\text { izquierda contra el substrato. }\end{array}$ & $0: 17$ & $<1$ \\
\hline 6 & $\begin{array}{l}\text { Limpieza de pico } \\
\text { con la pata }\end{array}$ & $\begin{array}{l}\text { El ave se limpia el pico con la pata, } \\
\text { levanta la pata y la dirige al pico para su } \\
\text { limpieza. }\end{array}$ & $0: 47$ & 2.6 \\
\hline 7 & Acomodo de alas & $\begin{array}{l}\text { El ave da una pequeña sacudida de su } \\
\text { cuerpo para poder acomodar las plumas } \\
\text { de su ala. }\end{array}$ & $0: 17$ & $<1$ \\
\hline 8 & $\begin{array}{l}\text { Erección de } \\
\text { plumas }\end{array}$ & $\begin{array}{l}\text { El ave se encuentra parada y da un giro } \\
\text { brusco de su cuerpo en el mismo lugar } \\
\text { permitiendo el erizamiento de las plumas. }\end{array}$ & $0: 38$ & 2.1 \\
\hline 9 & $\begin{array}{l}\text { Movimiento de } \\
\text { cola }\end{array}$ & $\begin{array}{l}\text { El ave mueve la cola arriba y abajo } \\
\text { parado en el mismo lugar dando un } \\
\text { acomodo de las plumas de la cola. }\end{array}$ & 2:09 & 7.2 \\
\hline 10 & Sacude el cuerpo & $\begin{array}{l}\text { Aquí el ave da una sacudida fuerte de su } \\
\text { cuerpo. }\end{array}$ & $1: 03$ & 3.5 \\
\hline 11 & $\begin{array}{l}\text { Fricción de la } \\
\text { cabeza con el } \\
\text { pecho }\end{array}$ & $\begin{array}{l}\text { La cabeza la comienza a sobar con su } \\
\text { pecho lateralmente. }\end{array}$ & $0: 11$ & $<1$ \\
\hline
\end{tabular}


Cuadro 1. Continuación.

\begin{tabular}{|c|c|c|c|c|}
\hline No. & $\begin{array}{c}\text { Conductas } \\
\text { en nueve } \\
\text { categorías de } \\
\text { comportamiento }\end{array}$ & Caracterización de cada conducta & $\begin{array}{c}\text { No. horas de } \\
\text { observación }\end{array}$ & $\begin{array}{c}\text { Tiempo } \\
\text { total de } \\
\text { observación } \\
(\%) \\
\end{array}$ \\
\hline 12 & Estira las alas & $\begin{array}{l}\text { El Huerequeque extiende su(s) ala(s) para } \\
\text { darse posiblemente un aireado a las axilas } \\
\text { o a la parte ventral de su cuerpo. }\end{array}$ & $0: 11$ & $<1$ \\
\hline 13 & Estira la pata & $\begin{array}{l}\text { El ave estira la pata a veces juntamente } \\
\text { con el ala o también independientemente, } \\
\text { la extiende totalmente, esto se da después } \\
\text { de un descanso. }\end{array}$ & $0: 47$ & 2.6 \\
\hline 14 & $\begin{array}{l}\text { Rasca la cabeza } \\
\text { con la pata }\end{array}$ & $\begin{array}{l}\text { Aquí la pata va a rascar o acomodar la } \\
\text { pluma de la cabeza. }\end{array}$ & $1: 16$ & 4.2 \\
\hline 15 & Recojo de cera & $\begin{array}{l}\text { El ave dirige su pico hacia la cola para } \\
\text { poder recoger de ella la secreción de } \\
\text { la glándula uropigial, y así lubricar las } \\
\text { plumas de su cuerpo y de paso acomodar } \\
\text { sus plumas. }\end{array}$ & $0: 38$ & 2.1 \\
\hline \multirow[t]{2}{*}{16} & Sacude cabeza & $\begin{array}{l}\text { Realiza un movimiento de sacudida en su } \\
\text { cabeza, moviéndola para varios lados sin } \\
\text { moverse el del sitio. }\end{array}$ & $0: 16$ & $<1$ \\
\hline & Locomoción & & & \\
\hline 17 & Saltar & $\begin{array}{l}\text { El ave da saltos sobre su propio lugar, } \\
\text { extiende las alas y da un pequeño salto, } \\
\text { en el mismo sitio donde se dio el salto, no } \\
\text { hay distancia recorrida horizontalmente. }\end{array}$ & 0:02 & $<1$ \\
\hline 18 & Correr & $\begin{array}{l}\text { Recorre tramos corriendo a veces con el } \\
\text { ala pegado a su cuerpo otras con el ala } \\
\text { separada del mismo. }\end{array}$ & $0: 29$ & 1.6 \\
\hline 19 & Caminar & $\begin{array}{l}\text { El ave realiza caminatas yendo de un } \\
\text { punto donde hay aves juntas a otro grupo, } \\
\text { ubicándose cerca de estos, también solo } \\
\text { caminatas a cualquier punto. }\end{array}$ & $3: 16$ & 10.9 \\
\hline 20 & Vuelo corto & $\begin{array}{l}\text { Esta conducta se basa en un vuelo que } \\
\text { dura poco tiempo y no cubre gran terreno } \\
\text { la distancia recorrida. }\end{array}$ & 0:05 & $<1$ \\
\hline 21 & Vuelo largo & $\begin{array}{l}\text { Esta conducta se basa en un vuelo que } \\
\text { dura largo tiempo y cubre gran terreno la } \\
\text { distancia recorrida. }\end{array}$ & $0: 11$ & $<1$ \\
\hline
\end{tabular}


Cuadro 1. Continuación.

\begin{tabular}{|c|c|c|c|c|}
\hline No. & $\begin{array}{c}\text { Conductas } \\
\text { en nueve } \\
\text { categorías de } \\
\text { comportamiento }\end{array}$ & Caracterización de cada conducta & $\begin{array}{l}\text { No. horas de } \\
\text { observación }\end{array}$ & $\begin{array}{c}\text { Tiempo } \\
\text { total de } \\
\text { observación } \\
(\%)\end{array}$ \\
\hline \multicolumn{5}{|c|}{ Alimentación } \\
\hline 22 & Comer & $\begin{array}{l}\text { El ave da picotazos al suelo en búsqueda } \\
\text { de alimento, buscando semillas o insectos, } \\
\text { a veces escarba el suelo para buscar a sus } \\
\text { presas. }\end{array}$ & $0: 32$ & 1.8 \\
\hline \multicolumn{5}{|c|}{ Descanso } \\
\hline 23 & Pata levantada & $\begin{array}{l}\text { Es un tipo de descanso donde el ave esta } \\
\text { descansando con una pata levantada, } \\
\text { permaneciendo un tiempo así. }\end{array}$ & 0:04 & $<1$ \\
\hline 24 & Sentado & $\begin{array}{l}\text { Otra forma de descanso es cuando } \\
\text { el ave se sienta y toma un descanso } \\
\text { permaneciendo un tiempo considerable. }\end{array}$ & $1: 35$ & 5.3 \\
\hline \multicolumn{5}{|c|}{ Alerta } \\
\hline 25 & $\begin{array}{l}\text { Orientando la } \\
\text { cabeza }\end{array}$ & $\begin{array}{l}\text { En esta categoría esta en completo estado } \\
\text { de alerta, para ello mueve la cabeza } \\
\text { lateralmente o hacia delante y hacia atrás, } \\
\text { tratando de percibir algún peligro cercano. }\end{array}$ & $1: 03$ & 3.5 \\
\hline 26 & Centinela & $\begin{array}{l}\text { Aquí el ave gira el cuerpo tratando de } \\
\text { cubrir mayor espacio para estar más } \\
\text { atento ante cualquier peligro. }\end{array}$ & $7: 26$ & 24.8 \\
\hline \multicolumn{5}{|c|}{ Sonora } \\
\hline 27 & Grito de aviso & $\begin{array}{l}\text { Las aves emiten sonidos fuertes al inicio y } \\
\text { durante los vuelos largos. }\end{array}$ & $0: 03$ & $<1$ \\
\hline \multirow[t]{2}{*}{28} & Grito crepuscular & $\begin{array}{l}\text { La realizan cuando está oscureciendo } \\
\text { juntándose en pequeños grupos para } \\
\text { emitir sonidos, luego echan a volar y } \\
\text { realizan su actividad nocturna. }\end{array}$ & 0:06 & $<1$ \\
\hline & \multicolumn{4}{|l|}{ Defecación } \\
\hline \multirow[t]{2}{*}{29} & Defecación & $\begin{array}{l}\text { Retrae ligeramente las alas hacia arriba, } \\
\text { luego excretan las heces. }\end{array}$ & 0:05 & $<1$ \\
\hline & Social agonístico & & & \\
\hline 30 & $\begin{array}{l}\text { Persecución o } \\
\text { ataque de un } \\
\text { individuo a otro }\end{array}$ & $\begin{array}{l}\text { Ocurre a la hora crepuscular, un individuo } \\
\text { del chorlo cabezón divisa a otro, } \\
\text { posteriormente el primero con las alas } \\
\text { abiertas corre de lado a lado en zigzag } \\
\text { persiguiendo al segundo. }\end{array}$ & 0:02 & $<1$ \\
\hline
\end{tabular}


Cuadro 1. Continuación.

\begin{tabular}{|c|c|c|c|c|}
\hline No. & $\begin{array}{c}\text { Conductas } \\
\text { en nueve } \\
\text { categorías de } \\
\text { comportamiento }\end{array}$ & Caracterización de cada conducta & $\begin{array}{l}\text { No. horas de } \\
\text { observación }\end{array}$ & $\begin{array}{c}\text { Tiempo } \\
\text { total de } \\
\text { observación } \\
(\%) \\
\end{array}$ \\
\hline \multicolumn{5}{|c|}{$\begin{array}{c}\text { Social no } \\
\text { agonístico }\end{array}$} \\
\hline 31 & $\begin{array}{l}\text { Agrupamiento- } \\
\text { aproximación }\end{array}$ & Agrupación de las aves durante el día. & $0: 03$ & $<1$ \\
\hline 32 & Separación & $\begin{array}{l}\text { Ocurre cuando anochece, es entonces } \\
\text { que se retiran volando para realizar sus } \\
\text { actividades nocturnas. }\end{array}$ & 0:02 & $<1$ \\
\hline
\end{tabular}

podría ser debido a que el etograma incluyó solo una hora de observación crepuscular-nocturna (la cual fue en la última hora de sesión, entre las 18:00 a 19:00 horas), $\mathrm{y}$ el Huerequeque es un ave de comportamiento principalmente nocturno. El número promedio de conductas de comportamiento que se observaron por sesión en el Huerequeque fue de 13.6 (rango de 8-17).

\section{DISCUSIÓN}

El acicalamiento es la categoría del comportamiento que presentó el mayor número de conductas identificadas (16) (Cuadro 1). El acicalamiento consiste en cualquier actividad de cuidado o atención de la superficie corporal en animales (Iannacone 2006, Nonato-Mamani \& Iannacone 2011); también ha sido considerado como expulgamiento o despiojado (Weeks et al. 1995, Mooring et al. 2004). El acicalamiento es un comportamiento de aseo caracterizado por una secuencia de movimientos que suceden en forma cíclica (Hogan-Warburg et al. 1995, Tingle et al. 2003). Se ha demostrado que el acicalamiento reduce eficientemente la carga parasitaria y es una importante medida de control y línea de defensa contra los ectoparásitos que se transfieren directamente con un alto costo energético en el ave hospedera (Spruijt et al. 1992, Maier 2001). El mayor número de repertorios de conductas de acicalamiento podría ser un indicador de que las aves están estresadas, tal como sugieren Moyer et al. (2003) y Henson et al. (2011). Esto puede ocurrir quizás por la presencia de la actividad humana en el área de estudio, en especial al sur por el personal de administración del local del CIAE y en algunas ocasiones por el tránsito en el borde interno del CIAE de algunos estudiantes y docentes universitarios. Por otro lado, se ha observado que un aumento en el acicalamiento de Larus glaucescens se ha relacionado con altas humedades y bajas temperaturas ambientales (Henson et al. 2007). De igual forma el alto porcentaje en el comportamiento de acicalamiento en relación al tiempo total de observación y el alto número de conductas en el acicalamiento de B. superciliaris 

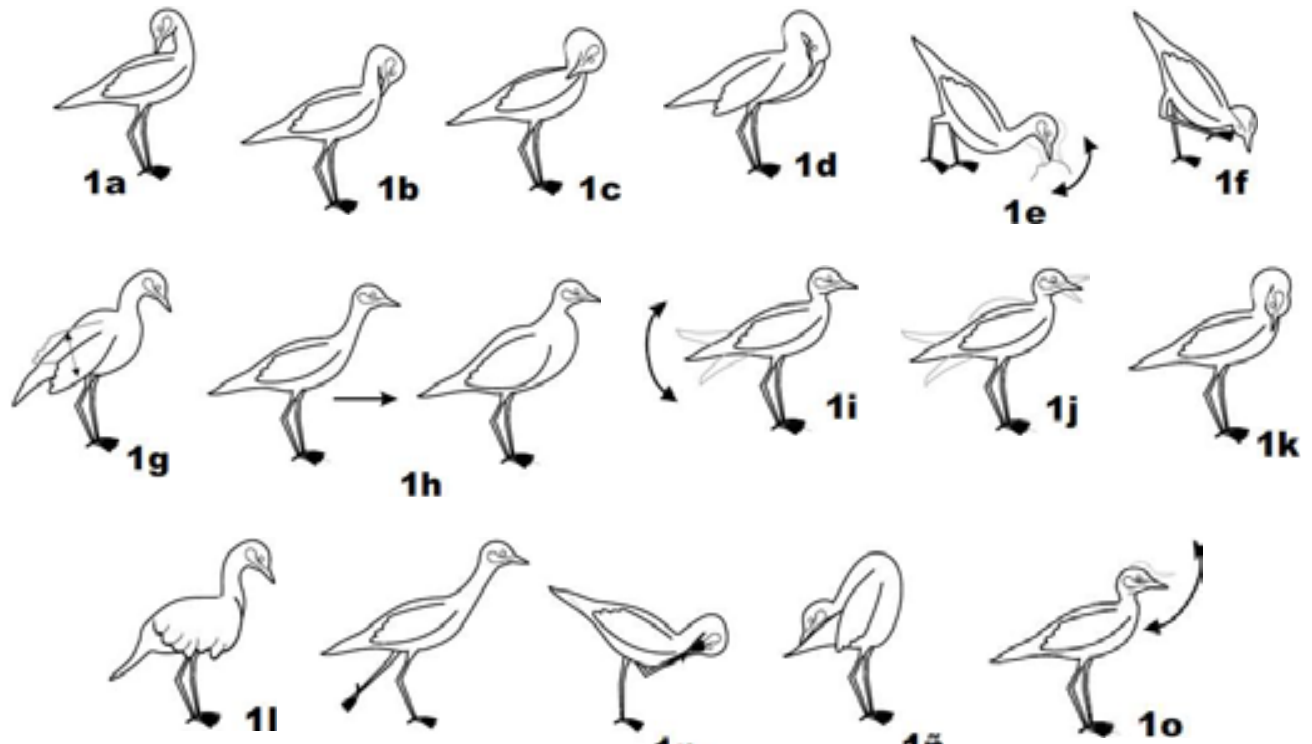

$1 \mathrm{~m}$

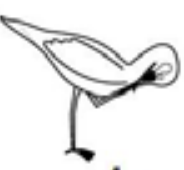

$1 \mathrm{n}$

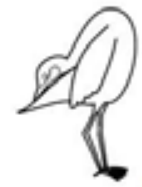

$1 \tilde{\mathbf{n}}$

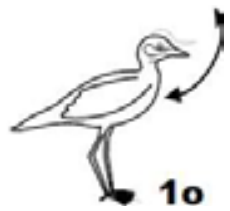

10

Figura 1. Limpieza de las plumas del dorso: (a); Limpieza de las plumas de pecho: (b); Limpieza de las plumas de las alas: (c); Limpieza de las plumas de las axilas: (d); Limpieza de pico: (e); Limpieza de pico con la pata: (f); Acomodo de alas: (g); Erección de plumas: (h). Movimiento de la cola: (i); Sacude el cuerpo: (j); Fricción de la cabeza con el pecho: (k); Estira las alas: (1); Estira la pata: (m); Rasca la cabeza con la pata: (n); Recojo de cera: (ñ). Sacude la cabeza: (o).

podría relacionarse con una alta humedad relativa de $85 \%$-91\% y una disminución de temperatura de $16.9 \pm 2{ }^{\circ} \mathrm{C}$ durante mayo a julio del 2006 en la zona del Callao, lo cual permitiría mantener en adecuadas condiciones el plumaje como un aislamiento térmico. El acicalamiento también ayuda a enfrentar a las aves a una situación de estrés como los depredadores y a un aumento de la intensidad de sonido, y otras actividades antrópicas (Henson et al. 2011). El acicalamiento es un mecanismo de alivio o confort ante el estrés y para eliminar olores que pudieran atraer los potenciales depredadores (tales como el cernícalo, la lechuza de los arenales y quizás los perros empleados como vigilantes del local del CIAE), los cuales han sido observados en algunas ocasiones persiguiendo al huerequeque por el personal administrativo del CIAE. El acicalamiento se ha encontrado como un comportamiento predominante en el etograma de otras especies de aves (Wiens et al. 1987, Martrechar et al. 1999), como en los Charadriformes Rynchops niger y Recurvirostra americana (Boettcher et al. 1994, Branco \& Fracasso 2005), ambas del mismo orden taxonómico que el Huerequeque. La especie congenérica Burhinus oedicnemus emplea un alto porcentaje de horas del día en el acicalamiento (Karavaev 1998). Por el contrario, McNeil et al. 

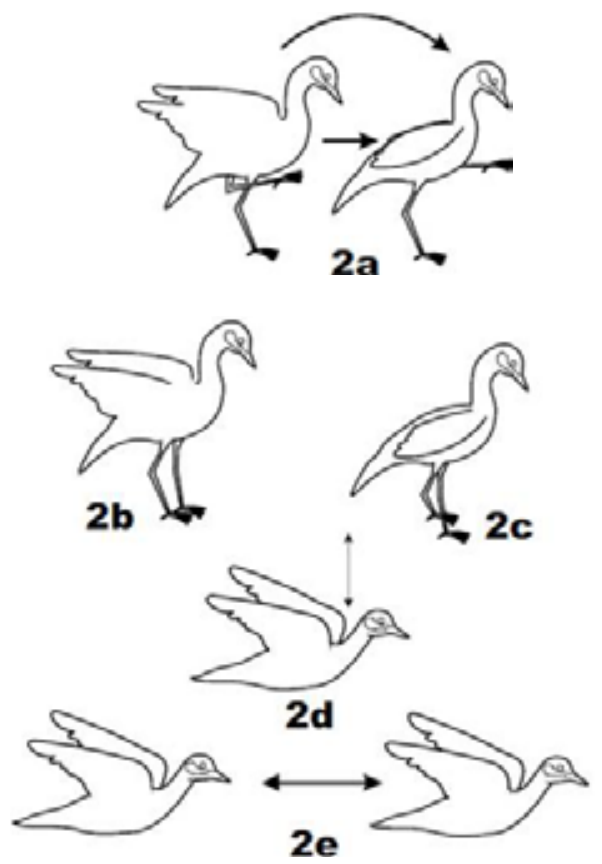

Figura 2. Saltar: (a); Correr: (b); Caminar: (c); Vuelos cortos: (d); Vuelos largos: (e).

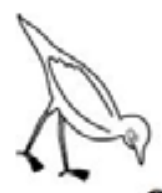

Figura 3. Comer: (22).

(1992) encontraron para otras especies de Charadriiformes nocturnas en humedales naturales que los comportamientos predominantes en el día eran el alimentarse (47$70 \%$ ) y el descanso (7-37\%) y que muy poco tiempo empleaban en otras actividades, como el acicalamiento. En otras aves como en Rhea americana los comportamientos de caminar y alerta fueron los dos más observados, al parecer debido a que el foco principal de ese estudio fue evaluar en forma experimental en cautiverio el efecto anti-depredador (Azevedo \& Young 2006). En el presente estudio, las categorías de comportamiento con mayor tiempo de observación de conductas por alerta (indicador de estrés) y por locomoción (mayormente indicador de relajamiento) fueron segunda y tercera en importancia, después del acicalamiento. 

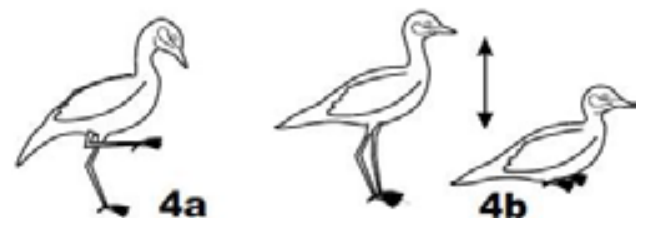

Figura 4. Pata levantada: (a); Sentado: (b).
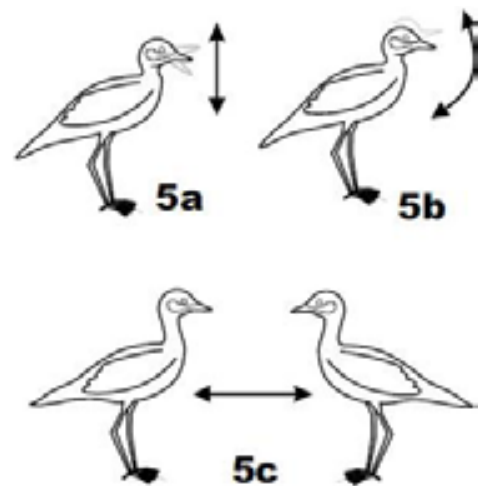

Figura 5. Orientando la cabeza: (a,b); Centinela: (c).

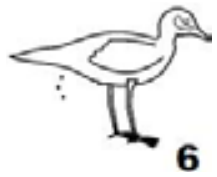

Figura 6. Defecación: (a).

Dentro de la categoría alerta la conducta centinela o vigilancia es el movimiento que más realizó $B$. superciliares durante las horas de estudio. Esto quizás podría deberse a que la zona de estudio se encuentra dentro de un área antropizada con agroecosistemas y vegetación xerofítica, y también a que es una conducta indicadora de estrés al quedarse en alerta durante periodos largos de tiempo (Porto \& Piratelli 2005). Salinas et al. (2003) indican que $B$. superciliares es un ave sensible a la presencia humana, pues se restringe a lugares desérticos con escasa o nula afluencia humana. Al parecer pocos meses después de 1997, año en que se inaugura oficialmente el ex Fundo Oquendo o Centro de investigaciones Agroecológicas de la UNFV, se asentaron las primeras poblaciones de B. superciliaris (Ventura et al., 2007). Maruyama et al. (2010) señalaron que el charadriiforme Vanellus chilensis para Minas 
a)

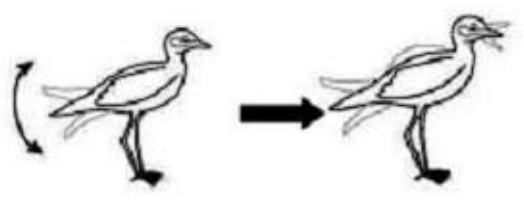

b)

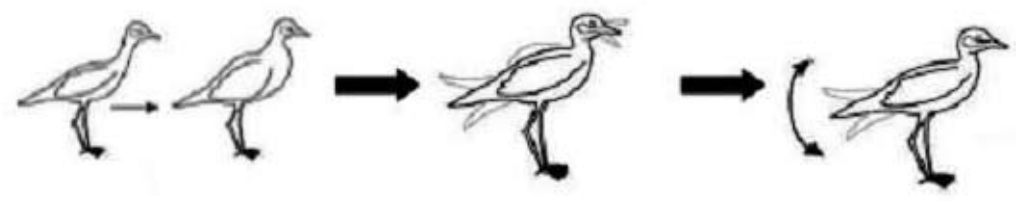

c)

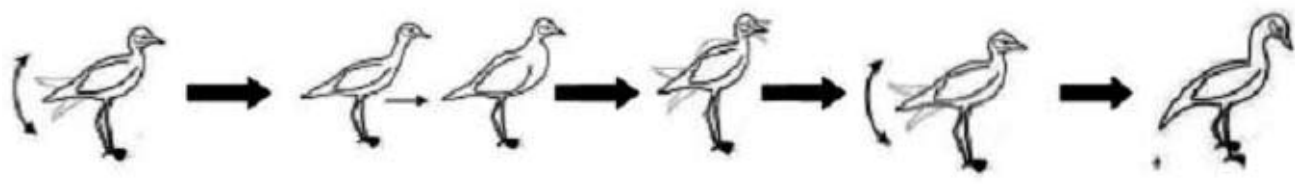

d)

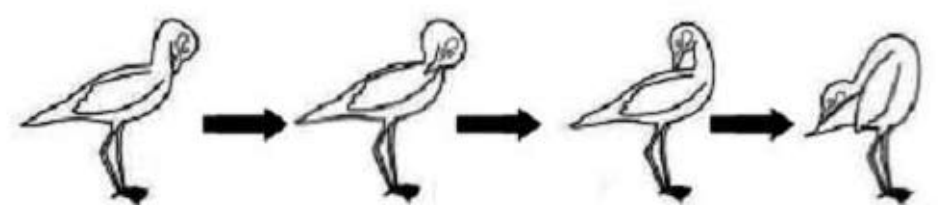

Figura 7. Etograma de conductas relacionadas en secuencia: a) movimiento de la cola - 1 y sacude el cuerpo - 2 (dos eventos) (>1 min); b) erección de plumas - 1, sacudido del cuerpo - 2 y movimiento de cola - 3 (tres eventos) (1 min); c) movimiento de la cola - 1, erección de plumas - 2, sacudida del cuerpo - 3, movimiento de cola - 4 y camina - 5 (cinco eventos) (1 min); d) limpieza de las plumas del pecho - 1, limpieza de las plumas del ala - 2, limpieza de las plumas del dorso - 3 y recojo de cera - 4 (cuatro eventos) (3 min aproximadamente).

Gerais, Brasil, presenta la conducta de centinela como la predominante en $100 \mathrm{~h}$ de observación, y lo asocia al aumento de disturbios por el hombre y los animales.

Las tres principales categorías de agrupación de comportamiento en orden porcentual decreciente con relación al tiempo total de observación fueron: acicalamiento $(49.7 \%)$, alerta $(28.3 \%)$ y locomoción $(13.5 \%)$. En $V$. chilensis la secuencia en orden decreciente fue diferente, alerta (31.67\%), locomoción (19.86\%) y acicalamiento (17.47\%) (Maruyama et al. 2010). Estas diferencias encontradas entre ambos estudios podrían ser atribuidas al grado de antropización de los ambientes donde se encuentran estas dos especies. 


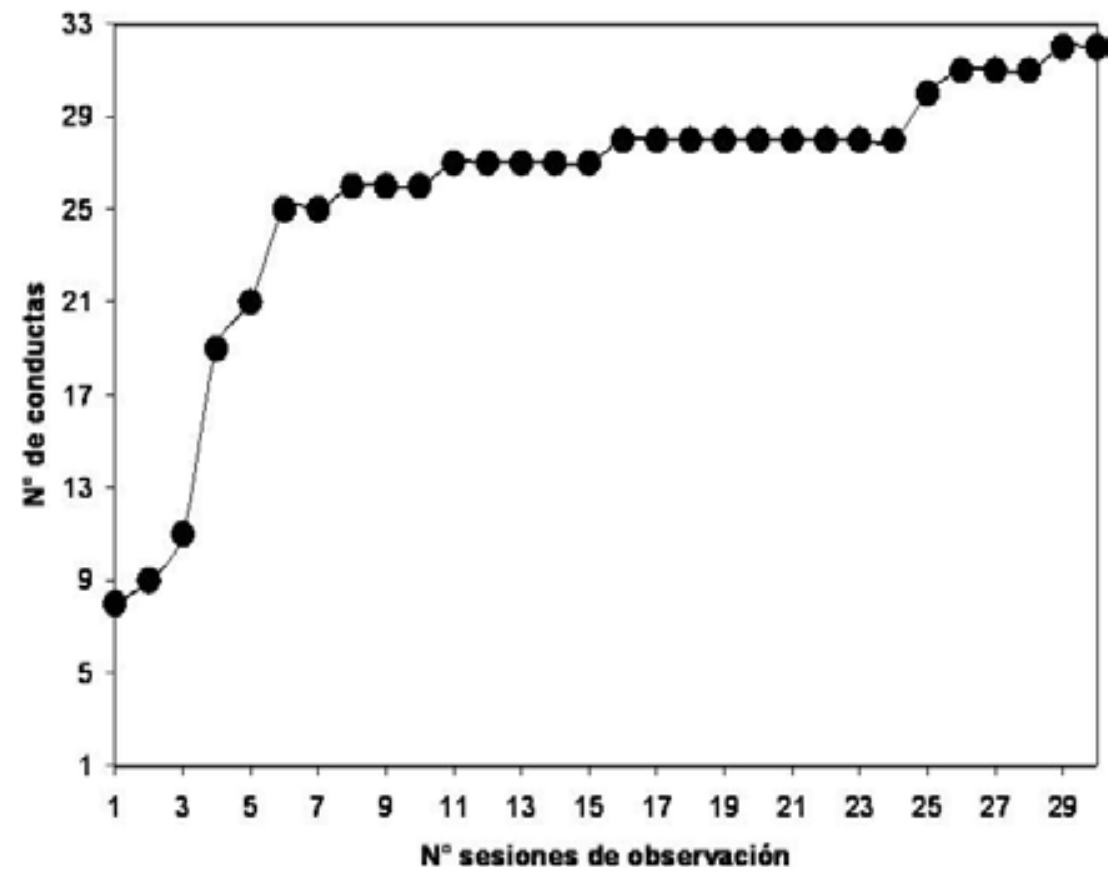

Figura 8. Curva de colecta para nuevas conductas de comportamiento de B. superciliaris en relación al número de horas (sesiones) de observación en Ventanilla, Callao, Perú.

Burhinus superciliaris es un ave de hábitos nocturnos y se ha visto que el tiempo de inactividad entre actividades es de alrededor de 3 a 4 min. Aunque en la zona se observaron escasamente otras aves y animales domésticos, no se evidenció ninguna interacción interespecífica relevante con las dos aves más predominantes y abundantes como la cuculí (Zenaida meloda) y la paloma doméstica (Columba livia), ni con perros. En las relaciones intraespecíficas se observaron comportamientos gregarios como pequeñas bandadas y agrupamientos o proximidad entre dos individuos. Una limitante del presente estudio para entender las relaciones fue que el registro del etograma de $B$. superciliaris no se realizó en las horas nocturnas de mayor actividad. Se denotó que esta ave comienza a aumentar su actividad en el crepúsculo como ha sido señalado para otras especies de Burhinus (Jones 1997). En otra ave charadriiforme nocturna $R$. americana, el repertorio completo de comportamientos visto durante el día también se observó en la noche, pero con diferencias en el tiempo empleado para cada actividad (Johnson et al. 2003, Kostecke \& Smith 2003). En las aves nocturnas Tringa flavipes, Calidris minutilla y Phalaropus tricolor, incrementan durante la noche el comportamiento de alimentación, por ser sus presas más accesibles y para evitar a los depredadores diurnos (Andrei et al. 2007). 
El tamaño de la bandada y el periodo del año evaluado (mayo a julio del 2006) que incluye las condiciones del clima como la temperatura, luminosidad, nubosidad, viento y hora de día (amanecer, crepúsculo o nocturno) determinan la cantidad y frecuencia de movimientos realizados por B. superciliaris (McNeil et al. 1992, Boettcher et al. 1994, Salinas et al. 2003, Andrei et al. 2007). Tres conductas de $B$. superciliaris como el grito crepuscular, la persecución de un individuo a otro y la separación ocurrieron exclusivamente en las horas crepusculares-nocturnas (Cuadro 1). Los patrones de comportamiento o etogramas del Huerequeque, que son parte de los estudios básicos de la biología de esta especie, pueden ser de utilidad para la elaboración de programas de monitoreo, planes de acción y estrategias regionales de conservación in situ bajo el sistema nacional de áreas protegidas en el Perú (SERNANP), principalmente en el Parque Nacional Cerros de Amotape, las Reservas Nacionales de Lachay, Paracas, Lagunas de Mejía, y los Refugios de vida silvestre Laquipampa y Pantanos de Villa (Astohuamán \& Espejo 2004). Lo anterior debido a que pudieran presentarse comportamientos anómalos, autodestructivos o estereotipados frente a situaciones ambientales inadecuadas como la destrucción, degradación y fragmentación de sus hábitats, o en muchos casos el incremento de ciertos comportamientos indicadores de estrés como el de alerta y el acicalamiento ante la presencia humana. En adición, es de interés continuar investigando los etogramas de conforte, locomotor, social, trófico y reproductivo de las aves debido a su utilidad en la determinación del horario más adecuado para los censos y para programas de reintroducción exitosos de especies en su ambiente natural (Gabriel \& Pizo 2005). Estudios previos con relación a patrones del comportamiento del Huerequeque son escasos (Camacho 2012). Por lo que es indispensable realizar estudios del comportamiento nocturno de esta ave.

El aporte fundamental de esta investigación sobre el comportamiento diurno de B. superciliaris es proporcionar información básica de patrones indicadores de relajamiento o de estrés a profesionales e investigadores de esta especie, para tomar medidas necesarias para favorecer su conservación in situ y ex situ.

Agradecimientos. A Sandra Cuadros, Sofía Espinoza, Marco Lovato, Flor Tello y Shirley Salazar por su apoyo logístico en el trabajo de campo. Los autores desean hacer constar su agradecimiento a los revisores anónimos por sus excelentes comentarios y sugerencias que han contribuido a mejorar la calidad de este artículo.

\section{LITERATURA CITADA}

Alcántara, B. A. 2010. Educación, turismo y recursos naturales de la región Callao. Revista del Instituto de Investigaciones FIGMMG, 13: 95-102.

Andrei, A.F., Smith, L.M., Haukos, D.A. \& Johnson, W.P. 2007. Behavior of migrant shorebirds in saline lakes of the Southern Great Plains. Waterbirds, 30: 326-334.

Aguirre, J., Demangel, R. \& Canepa, A. 2006. Apuntes sobre la nidificación del Chorlo Cabezón (Burhinus superciliaris) en el norte de Chile. Boletín Chileno de Ornitología, 12: 36-37. 
Iannacone et al.: Comportamiento de Burhinus superciliaris

Ângulo-Pratolongo, F., Schulenberg, T. S. \& Puse-Fernández, E. E. 2010. Las aves de los Humedales de Eten, Lambayeque, Perú. Ecología Aplicada, 9: 71-82.

Astohuamán, U. J. \& Espejo, J.M. 2004. Estudios preliminares de la distribución de aves en el área de Amortiguamiento de la Reserva Nacional de Paracas Pisco Playa- San Andrés, julio 2002-2003. Revista Cientifica Dilloniana, 4:105-107.

Azevedo, C. S. \& Young, R. J. 2006. Behavioural of captive-born Grater Rheas Rhea Americana Linnaeus (Rheiformes, Rheidae) submitted to antipredator training. Revista Brasileira de Zoología, 23: 186-193.

Birdlife International. 2004. Threatened birds of the world 2004. IUCN 2006. 2006 IUCN Red List of Threatened species. (En línea). Disponible en http:// www.iucnredlist. (Revisado el 17 de Agosto del 2006).

Blumstein, D.T. 2006. Developing an evolutionary ecology of fear: How life history and natural history traits affect disturbance tolerance in birds. Animal Behaviour, 71: 389-399.

Boettcher, R., Haig, S. M., William, C. \& Bridges Jr., W. C. 1994. Behavioral patterns and nearest neighbor distances among nonbreeding American Avocets. The Condor, 96: 973-986.

Branco, J. O. \& Fracasso, H. A. A. 2005. Ocurrência e abundância de Rynchops niger Linnaeus, no litoral de Santa Catarina, Brasil. Revista Brasileira de Zoología, 22: 430-432.

Camacho, C. 2012. Variations of flocking behaviour from core to peripheral regions of a bird species' distribution range. Acta ethologica, 15: 153-158.

Córdova-Rojas, W. M. \& Iannacone, J. 2011. Enriquecimiento ambiental para la disminución de conductas anómalas en el "Gato Montes" Leopardus colocolo (Molina, 1782) (Carnivora: Felidae) en un zoológico de Lima, Perú. Revista de Ciencias (Lima), 8: 17-37.

Emlen, S. T. \& Wrege, P. H. 2004. Division of labour in parental care behavior of a sex-role reversed shorebird, the Wattled Jacana. Animal behavior, 68: 847-855.

Gabriel, V.A. \& Pizo, M.A. 2005. Foraging behavior of Tyrant Flycatchers (Aves: Tyrannidae) in Brazil. Revista Brasileira de Zoología, 22: 1072-1077.

Griggio, M. \& Hoi, H. 2006. Is preening behaviour sexually selected? An experimental approach. Ethology, 112: 1145-1151.

Henson, S. M., Galusha, J. G., Hayward, J. L. \& Cushing, J. M. 2007. Modeling territory and preening in a seabird colony as functions of environmental conditions. Journal of Biological Dynamics, 1: 95-107.

Henson, S. M., Weldon, L. M., Hayward, J. L., Greene, D. J., Morgan, L. C. \& Screm, M. C. 2011. Coping behaviour as an adaptation to stress: post-disturbance preening in colonial seabirds. Journal of Biological Dynamics, 4: 1-21.

Hogan-Warburg, A. J., Hogan, J. A. \& Ashton, M. C. 1995. Locomotion and grooming in Crickets: Competition or time sharing?. Animal Behaviour, 49: 531-533.

Iannacone, J. 2006. Comportamiento de acicalamiento de adultos de "Mosca Domestica" Musca domestica L. (Diptera: Muscidae). Boletín de Lima (Perú), 143: 5-14.

Iannacone, J., Atasi, M., Bocanegra, T., Camacho, M., Montes, A., Santos, S., Zuñiga, H. \& Alayo, M. 2010. Diversidad de aves en el humedal Pantanos de Villa, Lima, Perú: periodo 2004-2007. Biota Neotropica, 10 (2), (En línea) Disponible en http//www.biotaneotropica.org.br/v10n2/es/abstract?/inventory+bn02610022010. (Revisado el 15 de enero del 2011).

Ibañez, L. M. \& Iannacone, J. 2011. Bioecología y estado de conservación del Condor de Selva Sarcoramphus papa Linnaeus, 1758 (Cathartiformes: Cathartidae): revisión a nivel de sudamérica. Biotempo, 11: 17-35.

Johnson, M., Beckmann, J. P. \& Oring, L.W. 2003. Diurnal and nocturnal behavior of breeding American Avocets. The Wilson Bulletin, 115: 176-185. 
Jones, J. 1997. Thick-Knee Studbook. North American Regional Studbook June 15, 1997. The Phoenix Zoo. AZ, USA. 12 p. + Anexos.

Karavaev, A. A. 1998. Daily activity of Stone Curlew Burhinus oedicnemus during the breeding period. International Wader Studies, 10: 329-332.

Kostecke, R. M. \& Smith, L. M. 2003. Nocturnal behavior of American Avocets in Playa wetlands on the Southern High Plains of Texas, USA. Waterbirds, 26: 192-195.

Lahitte, H. B., Ferrari, H. R. \& Lázaro, L. 2002. About the ethogram, 1: From the ethogram as a language to the language of ethogram. Revista de Etología, 2: 129-141.

Maier, R. 2001. Comportamiento Animal: Un enfoque evolutivo y ecológico. Mc.Graw-Hill/Interamericana de España, S.A.U. Madrid.

Martrenchar, A., Huonnic, D., Cotte, J. P., Boilletot, E. \& Morisse, J. P. 1999. Influence of stocking density on behavioral, health and productivity traits of Turkeys in large flocks. Britain Poultry Science, 40: 323-331.

Maruyama, P. K., Cunha, A. F., Tizo-Pedroso, E. \& Del Claro, K. 2010. Relation of group size and daily activity patterns to Southern Lapwing (Vanellus chilensis) behaviour. Journal of Ethology, 28: 339-344.

Mikich, S. B. 1991. Etograma de Ramphastos toco em cautiveiro (Piciformes: Rhampastidae). Ararajuba, 2: 3-17.

Miranda, L., Herreros De Lartundo, J. \& Knapton, B. 2001. Observaciones de Burhinus superciliaris en la Región de Tarapacá. Boletín Chileno de Ornitología, 8: 36.

Mooring, M. S., Blumstein, D. T. \& Stoner, J. C. 2004. The evolution of parasite-defense grooming in ungulates. Biological Journal of Linnean Society, 81: 17-37.

Moyer, B. R., Rock, A. N. \& Clayton, D. H. 2003. Experimental test of the importance of preen oil in rock doves (Columba livia). The Auk, 120: 490-496.

McNeil, R., Drapeau, P. \& Goss-Custard, J. D. 1992. The occurrence and adaptive significance of nocturnal habits in waterfowl. Biological Reviews, 67: 381-419.

Nonato-Mamani, L. \& Iannacone, J. 2011. Comportamiento del Oso de Anteojos Tremarctos ornatus (Cuvier, 1825) (Carnivora: Ursidae) en un zoológico de Lima, Perú. Revista de Ciencias (Lima), 8: 38-53.

Patraut, L. O. \& Riveros, J. C. 1998. Evaluación de la avifauna de los Pantanos de Villa, Lima. pp. 85103. In: Cano, A. \& Young, K. R. (Eds.). Los Pantanos de Villa Biología y Conservación. Museo de Historia Natural Serie de Divulgación $\mathrm{N}^{\circ} 11$. Lima, Perú.

Porto, G. \& Piratelli, A. 2005. Etograma da Maria-Preta, Molothrus bonariensis (Gmellin) (Aves, Emberizidae, Icterinae). Revista Brasileira de Zoologia, 22: 306-312.

Prestes, N. P. 2000. Descrição e análise quantitativa do etograma de Amazona petrei em cautiveiro. Ararajuba, 8: 25-42.

PromPeru (Comisión de Promoción del Perú para la Exportación y el Turismo). 2005. Guía Especializada del Viajero. Observación de la Naturaleza. PromPerú. Lima, Perú.

Rivadeneyra, F. J., Rivadeneyra, V. C. \& Mendoza, A. V. 2000. Comportamiento alimentario y reproductivo del Zarcillo Larosterna inca Lesson y Garnot, 1827 (Sternidae: Charadriiformes) en la Isla Pachacamac. Wiñay Yachay (Perú), 4: 7-26.

Salinas, L., Arana, C., Gavancho, L. \& Garrido, A. 2003. Distribución actual del Huerequeque (Burhinus superciliaris) en Lima. Revista Cientifica Dilloniana, 3: 97.

Salinas, L., Arana, C.\& Pulido, V. 2007. Diversidad, abundancia y conservación de aves en un agroecosistema del desierto de Ica, Perú. Revista peruana de biología, 13: 155-167.

Schulenberg, T. S., Stotz, D. E., Lane, D. F., O’Neill, P. J. \& Parker III, T. A. 2010. Aves de Perú. Serie Biodiversidad Corbidi (Centro de Ornitología y Biodiversidad), Surco, Lima, Perú. 
Spruijt, B. M., Van Hooff, J. A. R. A. M. \& Gispen, W. H. 1992. Ethology and neurobiology of grooming behaviour. Physiology Review, 72: 825-852.

Takano, G.F. \& Castro, I. N. 2007. Avifauna en el campus de la Universidad Nacional Agraria La Molina (UNALM), Lima, Perú. Ecología Aplicada, 6: 149-154.

Tingle, C. C., Rother, J. A., Dewhurst, C. F., Lauer, S. \& Kingm W. J. 2003. Fipronil: Environmental fate, ecotoxicology, and human health concerns. Review of Environmental Contamination and Toxicology, 176: 1-66.

Valqui, T. 2004. Where to watch birds in Peru. Ed. Team. Lima, Perú.

Vaz-Ferreira, R. 1984. Etología: El estudio biológico del comportamiento animal. Ed. Secretaría de la OEA. Washington, DC- EUA.

Ventura, K., Alegre, E., Chávez, K. \& Sócola, M. 2007. A la memoria del profesor Augusto Erwin Mendoza Valdivia (1958-2007). The Biologist (Lima), 5: 79-86.

Weeks, C. A., Nicol, C. J. \& Titchener, R. N. 1995. Effects of the Sucking Louse (Linognathus vituli) on the grooming behaviour of Housed Calves. The Veterinary Record, 137:33-35.

Wiens, J. A., Van Horne, B. \& Rotenberry, J. T. 1987. Temporal and spatial variations in the behavior of shrubsteppe birds. Oecologia, 73: 60-70.

Williams, M. D. 1981. Description of the nest and eggs of the Peruvian Thick-Knee (Burhinus superciliaris). The Condor, 83: 183-184. 